\title{
Solar turbulent magnetic fields: surprisingly homogeneous distribution during the solar minimum
}

\author{
L. Kleint ${ }^{1,2}$, S. V. Berdyugina ${ }^{3}$, A. I. Shapiro ${ }^{4}$, and M. Bianda ${ }^{2}$ \\ ${ }^{1}$ Institute of Astronomy, ETH Zurich, 8093 Zurich, Switzerland \\ e-mail: kleint@astro.phys.ethz.ch \\ 2 Istituto Ricerche Solari Locarno (IRSOL), 6605 Locarno Monti, Switzerland \\ 3 Kiepenheuer-Institut für Sonnenphysik, Schöneckstrasse 6, 79104 Freiburg, Germany \\ ${ }^{4}$ Physikalisch-Meteorologisches Observatorium Davos, World Radiation Center, Dorfstrasse 33, 7260 Davos Dorf, Switzerland
}

Received 25 June 2010 / Accepted 25 August 2010

\begin{abstract}
Context. Small-scale, weak magnetic fields are ubiquitous in the quiet solar atmosphere. Yet their properties and temporal and spatial variations are not well known.

Aims. We have initiated a synoptic program, carried out at the Istituto Ricerche Solari Locarno (IRSOL), to investigate both turbulent, mixed-polarity magnetic fields and nearly horizontal, directed fields and their variation with the solar cycle.

Methods. Through spectropolarimetric observations we monitor linear and circular polarization at the solar limb (5" on the disk) at five positional angles (N, NW, S, SW, W) with the sensitivity of $\sim 10^{-5}$. In addition, we analyzed measurements taken at different limb distances. We measure signatures in the $5141 \AA$ A region including two $\mathrm{C}_{2}$ triplets and three $\mathrm{Fe} \mathrm{I}$ lines. Linear polarization in these lines arises from scattering and can be modified via the Hanle effect in the presence of turbulent magnetic fields. Through the application of the differential Hanle effect to the $\mathrm{C}_{2} R$-triplet line ratios and the use of a simplified line formation model, we are able to infer a strength of turbulent magnetic fields while using the $P$-triplet to further restrict it. A Zeeman analysis of Fe I Stokes $V / I$ is used to evaluate flux densities of horizontally directed fields.

Results. We conclude that weak fields were evenly distributed over the Sun during this solar minimum. The turbulent field strength was at least $4.7 \pm 0.2 \mathrm{G}$, and it did not vary during the last two years. This result was complemented with earlier, mainly unpublished measurements in the same region, which extend our set to nearly one decade. A statistical analysis of these all data suggests that there could be a very small variation of the turbulent field strength ( $3 \sigma$-limit) since the solar maximum in 2000. The Zeeman analysis of Fe I Stokes $V / I$ reveals weak horizontal flux densities of 3-8 G.

Conclusions. Our results demonstrate the potential of long-term observations of small-scale magnetic fields, which may vary with the solar cycle in both mean strength and spatial distribution. This provides important constraints on the energy budget of the solar cycle. Extending this synoptic program to many spectral lines would provide a sample of heights in the solar atmosphere.
\end{abstract}

Key words. Sun: photosphere - polarization - scattering - magnetic fields - molecular processes

\section{Introduction}

Magnetic fields on the Sun appear in various sizes and shapes. While stronger, oriented fields can be detected via the Zeeman effect, very weak fields with different polarities below the resolution limit lead to a cancellation of the circularly polarized $(V / I)$ Zeeman signatures. It is believed that about $99 \%$ of the quiet Sun are governed by small-scale mixed-polarity magnetic fields, which renders their detection and characterization highly important (Stenflo et al. 1998).

The linear polarization signatures (Stokes $Q / I$ and $U / I$ ) arise either from transverse magnetic fields or from coherent scattering processes in the solar atmosphere. Anisotropic radiation, resulting from the temperature gradient, causes a net linear polarization usually parallel to the next solar limb. Atomic and molecular lines additionally polarize or depolarize the continuum polarization level, leading to a richly structured spectrum. This second solar spectrum (Ivanov 1991; Stenflo \& Keller 1997) gives us access to a wealth of information, among them an insight into the otherwise "hidden" turbulent magnetic fields.
They can be detected via the Hanle effect (e.g., Stenflo 1994), which usually causes a depolarization or a rotation of the plane of polarization in the presence of magnetic fields. Independently of the spatial orientation of the turbulent fields, the $Q / I$ spectrum usually gets depolarized, in contrast to signal cancellation in Zeeman measurements.

The interpretation of a single line in the second solar spectrum requires certain assumptions and constraints (radiative transfer with a model atmosphere, collision rates, etc.). However, the differential Hanle effect (Stenflo et al. 1998; Faurobert et al. 2001 ) is a very powerful tool. If several lines of the same species with the same formation height and different sensitivities to the magnetic field are observed in a single spectrum, many uncertain parameters can be eliminated. In particular, molecular lines provide a wide range of magnetic sensitivities and bear similar other properties (Berdyugina \& Solanki 2002; Berdyugina et al. 2002), and are therefore an excellent choice for the application of the differential Hanle effect. In addition, the filling factor of the magnetic field can be determined through this differential technique. 
Various measurements inferred turbulent fields with magnetic field strengths differing by one order of magnitude, between $10 \mathrm{G}$ and $100 \mathrm{G}$. This wide range can be caused by a variety of constraints involved into the interpretation. For instance, works based on the differential Hanle effect in molecular lines, i.e. largely model-independent, determined field strengths of 10-30 G (Faurobert et al. 2001; Faurobert \& Arnaud 2003; Berdyugina \& Fluri 2004; Derouich et al. 2006; Trujillo Bueno et al. 2006; Bommier et al. 2006). Those using a 3D model atmosphere analysis of the Sr I $4607 \AA$ line (Trujillo Bueno et al. 2004) concluded on a mean field strength of $130 \mathrm{G}$. To explain this drastic difference to molecular lines, they involved a hypothesis that scattering polarization in atomic and molecular lines form in different regions that are not yet spatially resolved by Hanle measurements. However, the field strengths obtained from a single atomic line should be considered as tentative upper limits, because modeling its depolarization involves an indefinite reference of the zero-field scattering polarization in the line, which is model-dependent.

Variations of the second solar spectrum during the solar cycle have been detected in strong atomic lines formed in the upper solar photosphere (Stenflo 2003), with the polarization amplitude generally decreasing during phases with stronger solar activity. For molecular lines, no spatial and temporal variations have been found so far, mainly because of their moderate polarization amplitudes and insufficient polarimetric sensitivity. Therefore, spatial and temporal variations of the second solar spectrum are still unclear and have never been thoroughly investigated.

We have initiated a synoptic observing program to investigate variations of the quiet Sun magnetic field. The molecular $\mathrm{C}_{2}$ region around $5141 \AA$ was chosen as the primary target because of its unique capability for the application of the differential Hanle effect (Berdyugina \& Fluri 2004) and, therefore, for studying variations of turbulent magnetic fields in the lower photosphere where these lines form. For fields in the upper atmosphere, two lines of the Cr I triplet at $5206 \AA$ and the Fe I $4383 \AA$ line were monitored and partly shown in Kleint et al. (2010a). Their analysis will be presented in a forthcoming paper. Simultaneously observed circular polarization is used to determine directed, line-of-sight magnetic fields at the solar limb in several lines with suitable Landé factors.

In this paper we present results of our synoptic observations of the $C_{2} 5141 \AA$ region for the period from December 2007 to December 2009, i.e. covering the solar minimum between the cycles 23 and 24. In Sect. 2 we describe the measurements and the data reduction procedure. We also include occasional observations in the same region from preceding years for completeness. In Sect. 3 we explain the differential Hanle effect, describe a simple model based on line ratios and address its advantages and limitations. The complete synoptic data set is analyzed in Sect. 4. We find no significant variations of the $\mathrm{C}_{2}$ Stokes $Q / I$ amplitude ratios during the solar minimum, but there may be a very small change as compared to the solar maximum. This implies possible mild variations of the average strength of the turbulent magnetic field during the solar cycle 23, as interpreted in Sect. 5.1. Modeling Stokes $V / I$ in Sect. 5.2 complements the information on the field distribution during the solar minimum. Finally, we discuss our results and summarize conclusions in Sect. 6. To address problems encountered with the line ratio model, we carry out an NLTE radiative transfer analysis of our

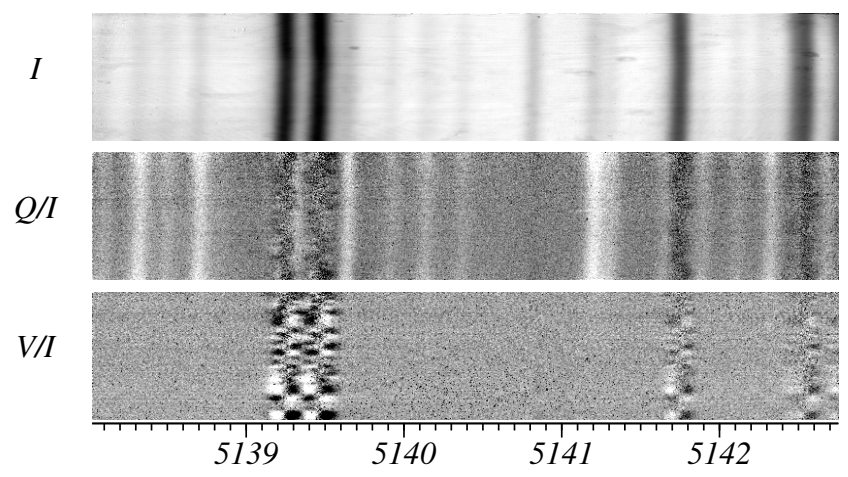

Fig. 1. Stokes $I, Q / I$ and $V / I$ spectra showing an example synoptic measurement taken on 16 August 2008 close to the heliographic south pole. $Q / I$ ranges from $-0.08 \%$ to $0.11 \%$ (with the continuum fixed at zero) and $V / I$ ranges from $-0.12 \%$ to $0.09 \%$.

data in a separate paper (Kleint et al. 2010b, hereafter Paper II). Yet this does not change our conclusions.

\section{Observations and data reduction}

\subsection{Synoptic program: ZIMPOL2 at IRSOL}

Our synoptic program for turbulent magnetic fields started in December 2007 before the appearance of the first sunspots of the solar cycle 24, and it is currently ongoing. Every two to three weeks, measurements of scattering polarization are taken in several selected wavelength regions, namely $\mathrm{C}_{2} 5141 \AA$, Cr I $5206 \AA$ and Fe I $4383 \AA$ at several position angles around the solar limb (N, NW, W, SW, S) near a heliocentric angle $\mu=\cos \theta$ $=0.1$ ( $5^{\prime \prime}$ from the limb) and with a slit of $0.5^{\prime \prime}$ width and $180^{\prime \prime}$ length. The observations are carried out with the UV sensitive ZIMPOL2 system (Gandorfer et al. 2004; Kleint et al. 2008) and the high-resolution spectrograph at the $45 \mathrm{~cm}$ telescope of the Istituto Ricerche Solari Locarno (IRSOL). The integration time of about $15 \mathrm{~min}$ is enough to achieve the sensitivity of $10^{-5}$, but together with telescope and camera overheads and calibrations each measurement for one single position angle and wavelength takes about $45 \mathrm{~min}$. Thus, we obtain one complete set of measurements during about two days. We carefully select regions of the quiet Sun and control it through simultaneous imaging in Stokes $V / I$, which ensures that no strong, oriented magnetic fields are in the field of view.

Simultaneously taken slit-jaw images were used to determine the limb distance, although a more accurate method was later applied using the scattering polarization amplitude of a $\mathrm{C}_{2}$ line with low magnetic sensitivity (see Sect. 4.2). The effect of detector nonlinearities, which scale with the incoming linear polarization offset, was significantly reduced through the implementation of a polarization compensation plate. This glass plate was used to compensate for the linear polarization produced by the telescope. It was placed immediately after the calibration optics and in front of the analyzer. Through a tilt and a rotation with respect to the optical axis, a linear polarization state can be created that is opposite to the telescope polarization, thus effectively canceling the polarization offset up to a level of $10^{-4}$.

A standard ZIMPOL data reduction (Gandorfer et al. 2004; Gisler 2005; Feller 2007) was employed, including darkframe correction, instrumental polarization calibrations and a crosstalk calibration. All $I / I_{\mathrm{c}}$ and $Q / I$ measurements were averaged along the slit for a higher signal-to-noise ratio, thereby lowering the 


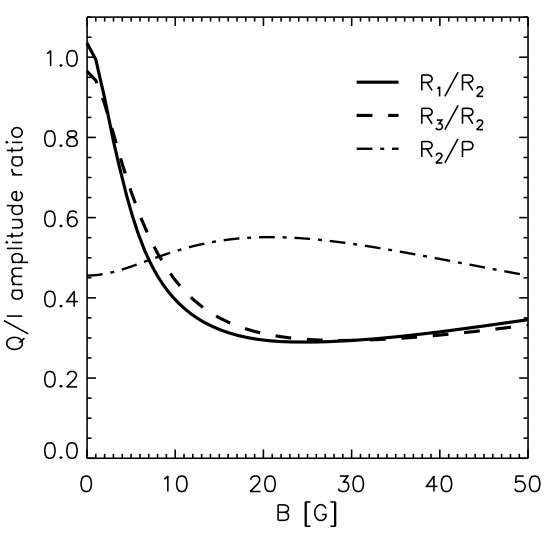

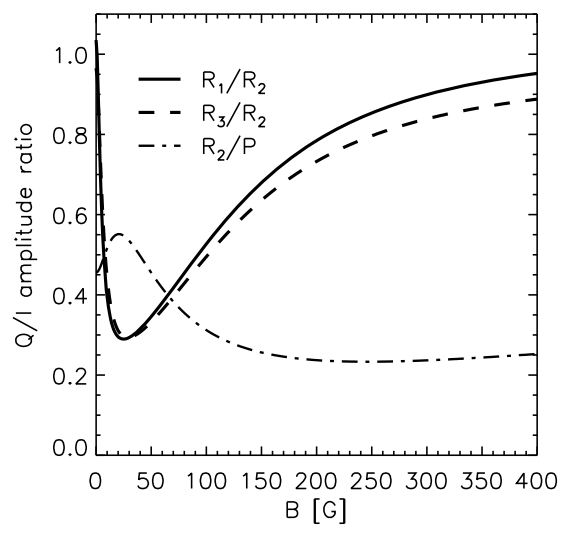

Fig. 2. Theoretical $Q / I$ amplitude ratios, calculated with the differential Hanle effect model. The left panel is a magnification of the right panel in the region from 0 to $50 \mathrm{G}$. spatial resolution to $180^{\prime \prime}$. The wavelength scale was determined by a cross-correlation of the observed $I$ spectra with the FTS atlas (Kurucz et al. 1984). The ZIMPOL system records three polarization states in one image (in our case $I Q V$ ), thus eliminating different pixel efficiencies in the polarized states $(Q / I, V / I)$. Because of the very fast modulation, seeing-induced crosstalk is also minimized. Flatfield images are not needed, because the polarized images only show an offset in the polarization level as compared to the flatfielded case, which is not important when using a differential analysis technique. However, Stokes $I$ should be treated with caution in our measurements.

Figure 1 shows an example of a calibrated record before averaging over the slit. Stokes $Q / I$ reveals several bright features (positive polarization above the continuum) with no apparent structure along the slit. Those mainly arise from molecular $\left(\mathrm{C}_{2}\right.$ and $\mathrm{MgH}$ ) lines. Darker features from strong atomic lines (depolarizing the continuum) show some spatial variations along the slit, which are most probably due to a weak transversal Zeeman effect. This is supported by Stokes $V / I$, which is very structured by the longitudinal Zeeman effect owing to small-scale, nearly horizontal magnetic fields that cannot be avoided even at the most quiet places on the Sun. Note that this is an exceptionally good measurement, while in general the structures in $V / I$ were smeared because of guiding errors or bad seeing. Typical circular polarization amplitudes amounted to $\pm 0.1 \%$ in atomic lines, while no Zeeman effect was seen in any of the molecular lines because of their small Landé factors.

\subsection{ZIMPOL2 at THEMIS}

Supplementary data were taken with ZIMPOL2 at the THEMIS telescope in June 2007 and June 2008, also at different positional angles near the solar limb. In addition, a very stable tip-tilt system at THEMIS allowed us to take a reliable sequence of measurements at different limb distances to investigate the center-tolimb variations and to determine accurately limb distances for measurements taken with other instruments. A polarization calibration unit was incorporated at THEMIS for these observing runs.

In addition, the data were corrected for field curvature resulting from the optics and flatfield effects. The polarized flatfield images taken at the solar disk center showed the telescope polarization offset and were subtracted from $Q / I$ and $V / I$. Theoretically, they would not be necessary at THEMIS either because of our differential analysis method.

\subsection{Earlier data}

To expand the time span of our measurement and, thus, to improve our search for temporal variations, we incorporated data from six MTR THEMIS observations from September 2004, two ZIMPOL2 IRSOL observations from July 2003 and the atlas of the second solar spectrum from 2000 by Gandorfer (2000). The older IRSOL data were taken with the ZIMPOL2 camera without micro-lenses. Therefore, they were obtained with longer integration times and still had a larger noise by about a factor of two compared to the current synoptic program.

The MTR data were reduced according to the procedure described in Bommier \& Rayrole (2002) and Bommier \& Molodij (2002). These data show severe fringes, which are varying with time and the polarization state. The fringes could not be removed with the flatfield images because the time difference between the observations was apparently too large. Neither FFT filtering nor fitting a combination of sine and cosine functions through several known "continuum points" significantly reduced the fringing. We therefore determined the $Q / I$ amplitudes for each spectral line with respect to nearby local continuum points which, according to recent measurements, are at the continuum polarization level. The error bars in the resulting MTR spectra are larger than in all other data sets, and systematic errors may be present.

A dataset from June 2006 obtained with MTR/THEMIS was presented by Asensio Ramos \& Trujillo Bueno (2007). They show the amplitudes of the lines to be larger by about a factor of two at the same limb distance $\mu$ when compared to our measurements with various instruments and telescopes. Also, the amplitudes of their measurements are not monotonically increasing as would be expected for each observation closer to the limb. We therefore cannot use their measurements for a comparison with our dataset.

\section{Differential Hanle effect in $\mathbf{C}_{2}$}

The region around $5141 \AA$ contains two $\mathrm{C}_{2}$ triplets, which are ideal for the application of the differential Hanle effect (Berdyugina \& Fluri 2004). The triplet $R_{1}(14), R_{2}(13)$ and $R_{3}(12)$ of the $R$ branch is prominent and unblended, and the $P$-triplet $P_{1}(42), P_{2}(41)$ and $P_{3}(40)$ is located next to the $R$-triplet and can be recorded in the same frame. These lines have a significantly different sensitivity to the Hanle effect, because their upper level Landé factors span two orders of magnitude (from 0.001 to 0.1 ), but their lifetimes are similar. They also form at about the same height in the solar atmosphere. 

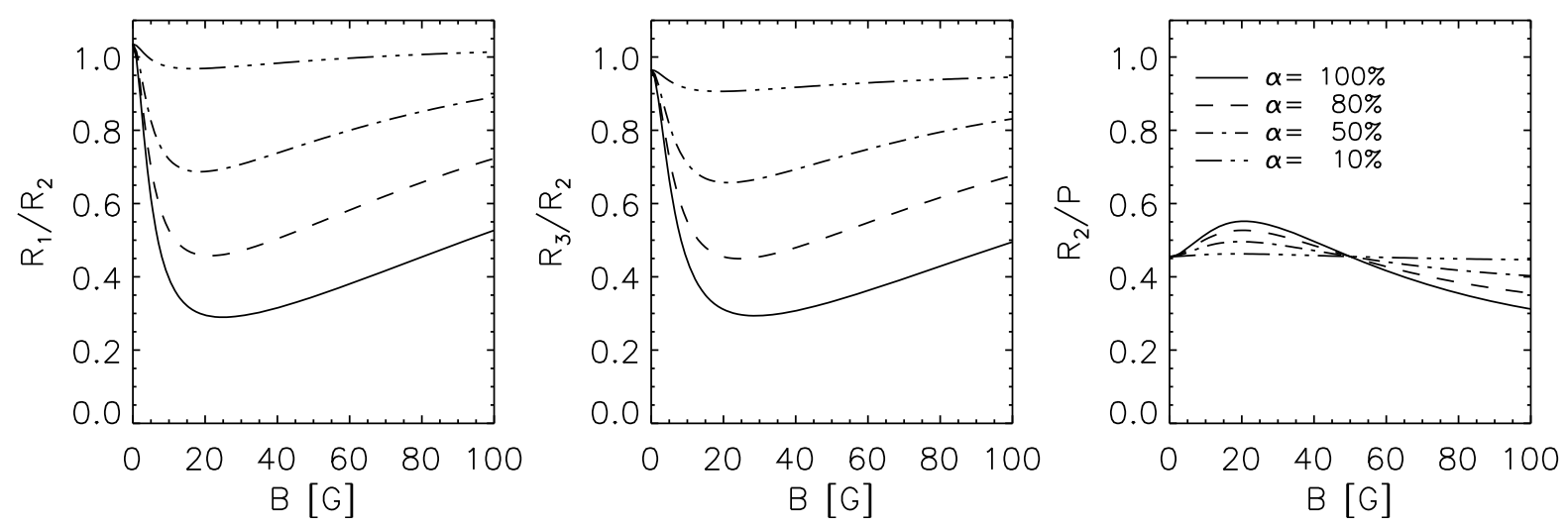

Fig. 3. $Q / I$ amplitude ratios for different values of the magnetic filling factor (solid: 1.0, dashed: 0.8 , dash-dotted: 0.5 , dash-three dots: 0.1 ).

Table 1. Physical parameters of our lines.

\begin{tabular}{lcccc}
\hline \hline Line & Wavelength $[\AA]$ & Landé factor & Lifetime $[\mathrm{s}]$ & $B_{\mathrm{C}}[\mathrm{G}]$ \\
\hline$R_{1}(14)$ & 5139.9258 & 0.13 & $1.23 \times 10^{-7}$ & 21 \\
$R_{2}(13)$ & 5140.1392 & 0.01 & $1.16 \times 10^{-7}$ & 231 \\
$R_{3}(12)$ & 5140.3760 & -0.12 & $1.07 \times 10^{-7}$ & 27 \\
$P_{1}(42)$ & 5141.2080 & 0.05 & $1.21 \times 10^{-7}$ & 58 \\
$P_{2}(41)$ & 5141.1909 & 0.002 & $1.18 \times 10^{-7}$ & 1622 \\
$P_{3}(40)$ & 5141.3081 & -0.05 & $1.15 \times 10^{-7}$ & 64 \\
\hline
\end{tabular}

The sensitivity of the line scattering polarization to the magnetic field can be characterized through a critical field $\left(B_{\mathrm{C}}\right)$ at which the Hanle effect is saturated and the line can no longer be depolarized. This is in contrast to the critical field $\left(B_{\mathrm{H}}\right)$ used by Trujillo Bueno (2003), where some depolarization is still possible. These two cases correspond to two different values of the Hanle depolarization parameter ( $W_{\mathrm{H}}$, see also Eq. (3)), namely

$W_{\mathrm{H}}=1-0.4\left(\frac{\gamma_{\mathrm{H}}^{2}}{1+\gamma_{\mathrm{H}}^{2}}+\frac{4 \gamma_{\mathrm{H}}^{2}}{1+4 \gamma_{\mathrm{H}}^{2}}\right)=\left\{\begin{array}{l}0.25 \text { for } B_{\mathrm{C}} \\ 0.48 \text { for } B_{\mathrm{H}},\end{array}\right.$

neglecting collisions. The parameter $\gamma_{\mathrm{H}}$ is given by the equation

$\gamma_{\mathrm{H}}=0.88 \times 10^{7} g_{\mathrm{L}} B t_{\text {life }}=\left\{\begin{array}{l}3.03 \text { for } B_{\mathrm{C}} \\ 1.00 \text { for } B_{\mathrm{H}},\end{array}\right.$

which leads to the two different possibilities for a critical field $B$. Below we will refer to the first case when we use the term "critical field". The critical field for the two $\mathrm{C}_{2}$ triplets ranges from $21 \mathrm{G}$ to $1622 \mathrm{G}$ assuming an isotropic field distribution. Their upper level Landé factors $g_{\mathrm{L}}$, lifetimes $t_{\text {life }}$ and $B_{\mathrm{C}}$ are given in Table 1.

Because the selected lines are optically thin, form at the same height, and are observed simultaneously, their polarization amplitude ratios can be employed to investigate turbulent magnetic fields, while eliminating many uncertainties related to the line formation processes in a model atmosphere (Berdyugina \& Fluri 2004). We calculate the ratios from the amplitudes above the local continuum level, i.e. with the continuum polarization shifted to the zero level. The amplitude ratios $R_{1} / R_{2}$ and $R_{3} / R_{2}$ are most sensitive to magnetic fields because $R_{2}\left(g_{\mathrm{L}}=0.01\right)$ has a significantly higher critical field than $R_{1}$ and $R_{3}\left(\left|g_{\mathrm{L}}\right|=0.1\right)$. Nevertheless, the $R$-triplet alone does not give a unique solution for the magnetic field strength, because the amplitudes of its components are almost equal for both very weak (few $G$ ) and strong (hundreds G) fields (see Fig. 2). In the second case, the lines become depolarized at the Hanle saturation level, and the amplitude ratios asymptotically approach unity again. Therefore, the $P$-triplet is needed as an additional reference. The $R_{2} / P$ ( $P$ denotes the total amplitude of $P_{1}$ and $P_{2}$ ) ratio helps to distinguish between weak $(<80 \mathrm{G})$ and strong regimes, with $g_{\mathrm{L}}=0.05$ and 0.002 for $P_{1}$ and $P_{2}$, respectively. The amount of depolarization of $P_{3}\left(\left|g_{\mathrm{L}}\right|=0.05\right)$ with respect to the $P$-peak also indicates the prevalent regime.

A simple model can be used to analyze weak lines with different magnetic sensitivities taken within one spectrum. Below we briefly describe the main principles of the model, while its detailed description can be found in Berdyugina \& Fluri (2004). To fit the observed Stokes $Q / I$ profiles, we used the same line parameters as in that paper, apart from the Einstein coefficients, which were reduced by a factor of two to satisfy our radiative transfer modeling of the same lines in Paper II and to abandon the previously assumed $\Lambda$-doubling, which had led to the higher coefficients.

The continuum of the solar $Q / I$ spectrum is linearly polarized through Thomson and Rayleigh scattering (Fluri \& Stenflo 1999; Stenflo 2006). Spectral lines can add polarization or depolarize the continuum. Further, collisions or magnetic fields can change (more often reduce) the line polarization by a degree depending on transition properties. For weak, optically thin lines, the depolarization of the continuum can be neglected, and the line polarization can be calculated independently of the continuum. In principle, scattering from continuum into the lines is also possible, but this effect is within the noise level of the data for our optically thin lines (Fluri \& Stenflo 2003). In our model, we set the continuum polarization to zero, calculate the scattering polarization for different molecular lines in a narrow spectral region and compose ratios of their amplitudes in order to eliminate unknown parameters. We assume a single value magnetic field, which fills a certain volume of the resolution element, characterized by the filling factor $\alpha$. This is the main difference to the model presented by Berdyugina \& Fluri (2004). We neglect collisions and assume the same formation height for all the observed $\mathrm{C}_{2}$ lines. The scattering polarization

$Q / I=\alpha \cdot q W_{\mathrm{H}} W_{\mathrm{c}} W_{2} k_{\Lambda v J} \Phi_{\lambda}+(1-\alpha) \cdot q W_{\mathrm{c}} W_{2} k_{\Lambda v J} \Phi_{\lambda}$

then depends on a Hanle depolarization factor $W_{\mathrm{H}}$, a collisional depolarization factor $W_{\mathrm{c}}$ (which is set to 1 in our case), the intrinsic line polarizability $W_{2}$, an opacity factor $k_{\Lambda v J}$ and the line profile $\Phi_{\lambda}$. The factor $q$ accounts for several factors of line formation, which are the same for all our lines and therefore cancel out when using the differential Hanle effect. To calculate actual 


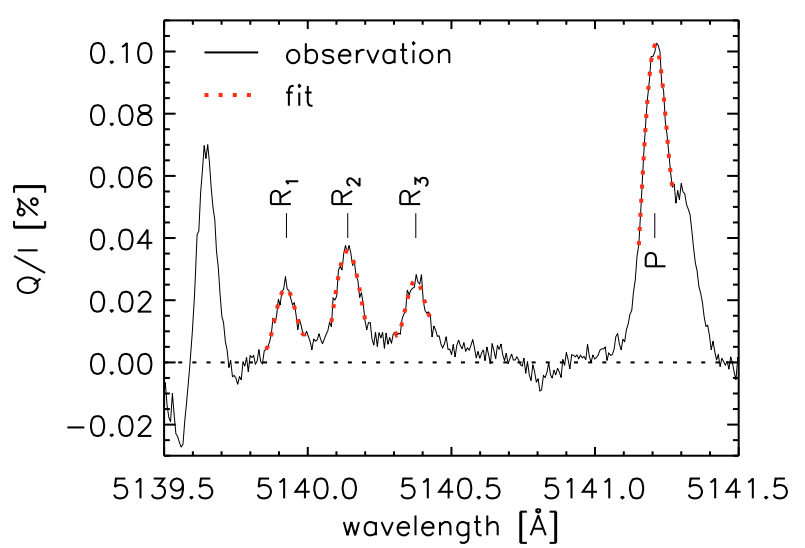

Fig. 4. Example fit of four polynomials (dotted) to an observation (solid) to be able to determine the amplitudes of the lines and their ratios.

line profiles $q$ can be used as a scaling factor. The amplitude ratios predicted by this model assuming $\alpha=1$ are shown in Fig. 2 . If at least two ratios are measured, the filling factor can be determined as well. Figure 3 shows its influence on the amplitude ratios. The lower the filling factor, the lower the changes in the ratios with increasing magnetic field. It would be possible to extend the analysis and use a continuous distribution of magnetic field strengths, for instance characterized through a probability distribution function (PDF). This is however beyond the scope of this simple model. We employ this model to interpret our Stokes $Q / I$ measurements in Sect. 5.1.

\section{Data analysis}

We calculated the amplitude ratios $R_{1} / R_{2}, R_{3} / R_{2}$ and $R_{2} / P$ for 102 synoptic measurements, all THEMIS observations and suitable older data. To determine these amplitude ratios all spectra were shifted to the same polarization level, which was fixed at zero for the average of the points between $5140.6 \AA$ and $5141.0 \AA$ ("continuum"). Then, a fourth-degree polynomial was fitted through the three $R$ peaks and the $P$ peak as demonstrated in Fig. 4 (dotted lines). These fits were used to calculate the ratios, therefore setting the continuum polarization to zero.

\subsection{Dependence on position angles}

Figure 5 shows the amplitude ratios of 78 synoptic measurements sorted by heliographic position angle (N, NW, S, SW, W). The observed position angles are not evenly distributed because of the exclusion of noisy measurements or weather constraints. The solid lines denote the averages per position angle, which are also presented in Table 2 with their standard deviations. Only the synoptic measurements with the limb distances $0.07<\mu<0.13$ are included in this plot in order to avoid a possible variation because of $\mu$. The error bars for the single older measurements in the table are derived from the residuals of the fits and their propagation into the ratios.

The noise level of the observations amounts to a maximum of about $5 \times 10^{-5}$, which is significantly lower than one standard deviation of the amplitude ratios. This implies that some local variations may have been present, because the used slit was quite long. If these variations are caused by a change in magnetic field strength, we can restrict them to be less than $\pm 1 \mathrm{G}$ (see Sect. 5.1). A possible local change in anisotropy or a variation of the filling
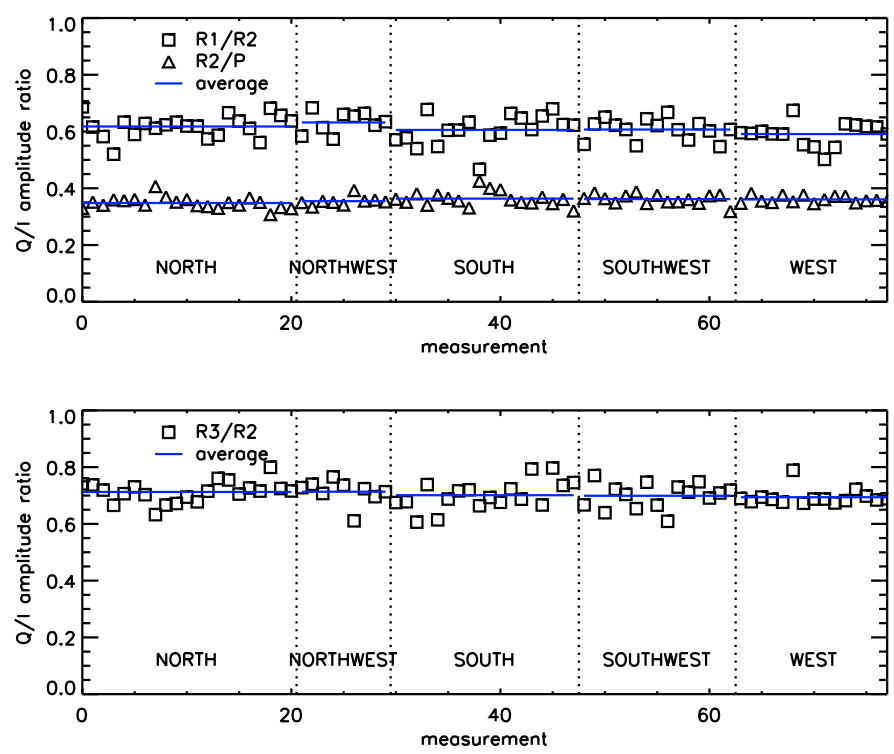

Fig. 5. $Q / I$ amplitude ratios for 78 synoptic measurements sorted by heliographic position angle. The ratios $R_{1} / R_{2}$ and $R_{2} / P$ are shown in the upper panel and $R_{3} / R_{2}$ in the lower panel. The solid lines show the averages per position angle. They are the same within one standard deviation for all angles. This indicates that the turbulent field is everywhere of equal strength.

Table 2. Average amplitude ratios for various datasets.

\begin{tabular}{lccc}
\hline \hline & $R_{1} / R_{2}$ & $R_{3} / R_{2}$ & $R_{2} / P$ \\
\hline IRSOL 2007-2009 & & & \\
$\mathrm{N}$ & $0.62 \pm 0.04$ & $0.71 \pm 0.04$ & $0.35 \pm 0.02$ \\
$\mathrm{NW}$ & $0.63 \pm 0.04$ & $0.71 \pm 0.04$ & $0.35 \pm 0.02$ \\
$\mathrm{~S}$ & $0.61 \pm 0.05$ & $0.70 \pm 0.05$ & $0.36 \pm 0.02$ \\
$\mathrm{SW}$ & $0.61 \pm 0.04$ & $0.70 \pm 0.04$ & $0.36 \pm 0.02$ \\
$\mathrm{~W}$ & $0.59 \pm 0.04$ & $0.69 \pm 0.03$ & $0.36 \pm 0.01$ \\
\hline THEMIS 2008 & $0.59 \pm 0.03$ & $0.73 \pm 0.03$ & $0.35 \pm 0.01$ \\
\hline THEMIS/MTR 2004 & $0.66 \pm 0.10$ & $0.62 \pm 0.10$ & $0.31 \pm 0.03$ \\
\hline IRSOL 2003 & $0.57 \pm 0.09$ & $0.71 \pm 0.08$ & $0.29 \pm 0.02$ \\
\hline Atlas 1999 & $0.52 \pm 0.05$ & $0.62 \pm 0.05$ & $0.43 \pm 0.02$ \\
\hline
\end{tabular}

factor would have a similar effect on the ratios and would diminish the variation of the magnetic field strength.

However, we see no systematic differences, neither between the position angles nor in time at a given position. We conclude therefore that the turbulent magnetic field has been of rather equal strength everywhere on the Sun during the past solar minimum at the depth of formation of the $\mathrm{C}_{2}$ lines.

There is only one measurement, taken on 9 May 2008 (hereafter $\mathrm{ms}_{080509}$ ) close to the south pole, which deviates from the rest by more than $3 \sigma$ in $R_{1} / R_{2}$ and $R_{2} / P$, but is close to the average in $R_{3} / R_{2}$. Its amplitude ratios are $R_{1} / R_{2}=0.47 \pm 0.03$, $R_{3} / R_{2}=0.66 \pm 0.04$ and $R_{2} / P=0.43 \pm 0.01$. This measurement will be discussed and compared with others in Sect. 4.3.

\subsection{Dependence on the limb distance}

The absolute amplitude of the $Q / I$ signal steeply decreases with increasing limb distance $\mu$. This may be attributed to a combination of depth-dependent anisotropy, collisional rates, or magnetic field and the scattering geometry. Indeed, the radiation emitted at higher $\mu$ forms deeper in the atmosphere where 
collisional rates are higher and therefore the contribution from scattering processes is lower.

The knowledge of the limb distance $\mu$ for every measurement is necessary for analyzing the data and for determining the magnetic field strength. However, the guiding system at IRSOL is precise to only about $1 \mathrm{arcsec}$ and therefore the uncertainty of the limb distance at $\mu=0.10$ is \pm 0.01 . Bad seeing or positioning can lead to an additional variation. A similar uncertainty came out of deriving $\mu$ from former slit-jaw images. Starting in April 2009, the slit-jaw imaging system at IRSOL was redesigned and improved and can now be used for continuous imaging every few seconds with a sufficiently high resolution. These slit-jaw images can later be averaged, providing an average $\mu$ and its variation during the observations.

For all older measurements, we employed the following method to determine an average limb distance. First, the measurements were ordered by ascending $\mu$ as given by the guiding program, keeping in mind that there may be errors up to a few arcseconds. Then, we used absolute amplitudes of the lines, which are weakly sensitive to the magnetic field as an indicator of $\mu$. In particular, the $R_{2}$ peak $\left(B_{\mathrm{C}}=231 \mathrm{G}\right)$ and the blend of the $P_{1}$ and $P_{2}$ peaks $\left(B_{\mathrm{C}}=58 \mathrm{G}\right.$ and $1622 \mathrm{G}$, respectively) are well-suited for this determination. A decrease of these amplitudes with $\mu$ can be attributed to changes in anisotropy and collision rates, but most probably not to the magnetic field.

Indeed, the center-to-limb variation of these peaks studied at both IRSOL and THEMIS was well reproducible at different limb distances. For example, our seven measurements at $\mu=0.1$ (4.8 arcsec from the nearest limb) at THEMIS 2008, each with a different position angle, using a tip-tilt adaptive optics system showed a $P$ amplitude of $0.106 \pm 0.005 \%$ and an $R_{2}$ amplitude of $0.036 \pm 0.003 \%$. For $\mu \geq 0.2$ a positioning error of 1 arcsec is not very critical and does not significantly influence the amplitudes. We found also that the center-to-limb variation of $Q / I$ of the $P$ and $R_{2}$ amplitudes obey the following semi-empirical relation from Stenflo et al. (1997) very well:

$\operatorname{amp}(\mu)=\frac{a \times\left(1-\mu^{2}\right)}{b+I_{\mathrm{c}}(\mu) \cdot \mu}$,

where $a$ and $b$ are free parameters. The values for the continuum intensity $I_{\mathrm{c}}(\mu)$ were taken from Allen (1973). According to Stenflo et al. (1997) this relation can be explained in the following way: weak molecular lines form in an optically thin layer, where the optical path length scales with $\mu$ as $\sim 1 / \mu$. Together with the source function for $Q$, which scales as $1-\mu^{2}$, this results in $Q \sim \frac{1-\mu^{2}}{\mu}$. For weak lines, Stokes $I$ can be approximated through the continuum intensity, which is strongly dependent on the limb distance and the wavelength: $I_{\mathrm{c}}(\mu, \lambda)$. This results in the proportionality of $Q / I \sim \frac{1-\mu^{2}}{I_{c}(\mu) \cdot \mu}$ for a given line. This relation also agreed well with their measured center-to-limb variations of molecular $\mathrm{MgH}$ lines. We fitted this relation to the $P$ and $R_{2}$ amplitudes measured at well-determined limb distances. Then we used it to derive more accurate $\mu$ values for those observations where slit-jaw images or guiding were unreliable.

The fit for the $P$ amplitude can be seen in Fig. 6. As expected, the error increases toward the limb, but it is still acceptable, so this method provides more precise $\mu$ values than the old slit-jaw images or the guiding program output. The fit for the $R_{2}$ peak amplitude (not shown in the figure) was of a slightly lower quality, because it was lower by about a factor of three than the $P$ peak, and small uncertainties in the determination of the $R_{2}$ amplitude lead to larger errors in the $\mu$ determination. However, for

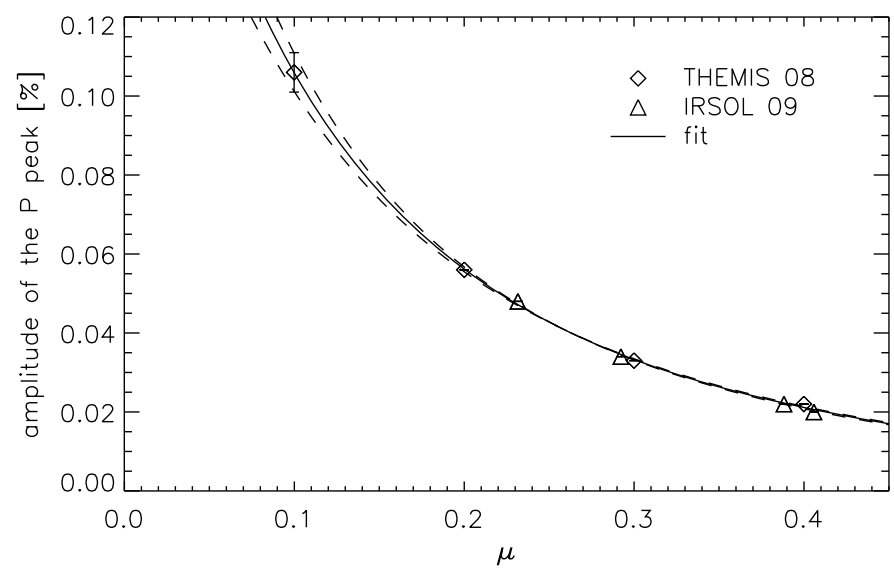

Fig. 6. Center-to-limb variation of the $P$ peak amplitude (diamonds and triangles for two sets of data). For $\mu=0.1$, seven measurements were averaged and their standard deviation is indicated. The solid line represents a fit using Eq. (4), and the dashed lines are fits using the $\mu=0.1$ amplitude with $\pm 1 \sigma$.
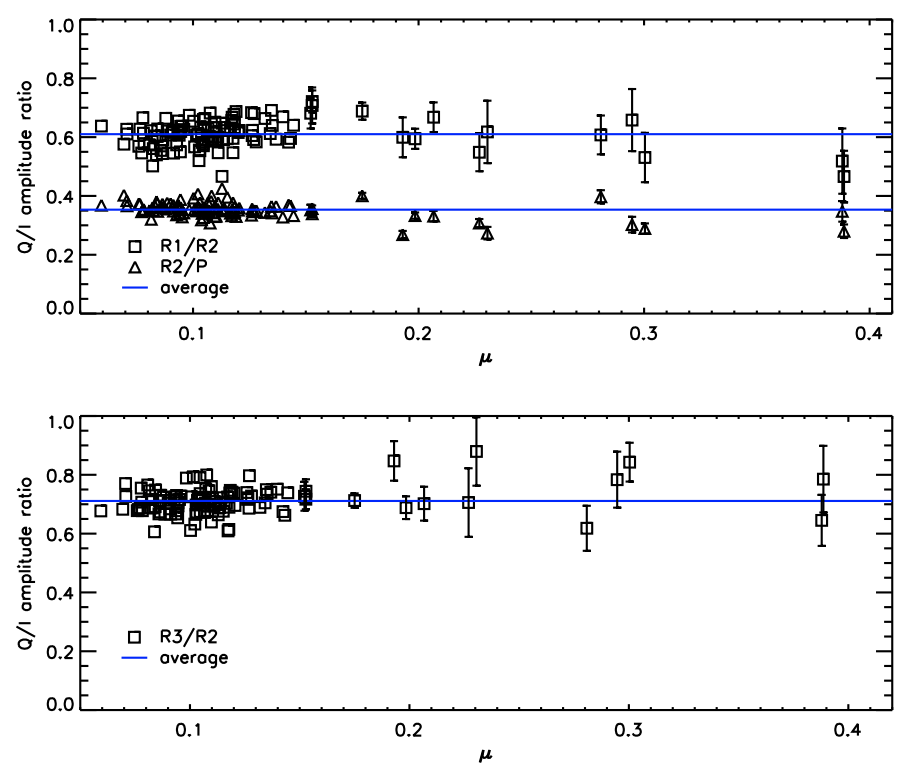

Fig. 7. $Q / I$ amplitude ratios vs. limb distance $\mu$. The solid lines represent the overall averages of all data points. For clarity reasons, the error bars are only drawn for measurements with $\mu>0.15$.

more than $75 \%$ of the cases $\mu$ values obtained from $P$ and $R_{2}$ differed by $<0.01$. Larger differences were generally for measurements with higher noise levels. Therefore, we chose the $\mathrm{P}$ amplitude to calibrate limb distances of the synoptic IRSOL measurements.

The amplitude ratios versus the calibrated limb distances are shown in Fig. 7 for all synoptic observations and some measurements at larger limb distances and for all position angles. The error bars for $R_{2} / P$ are smaller than the others because the signal-to-noise of the observations is higher for these peaks. Although the scatter about the average is relatively large, with $1 \sigma=0.04$, we can exclude systematic variations at higher limb distances larger than this scatter. This corresponds to the variations of the magnetic field with height of $<1 \mathrm{G}$ across the line formation region of about $100 \mathrm{~km} . R_{2} / P$ may be decreasing very slightly with increasing limb distance. There may be some fluctuations, but systematic errors may be present as well. $R_{3} / R_{2}$ 

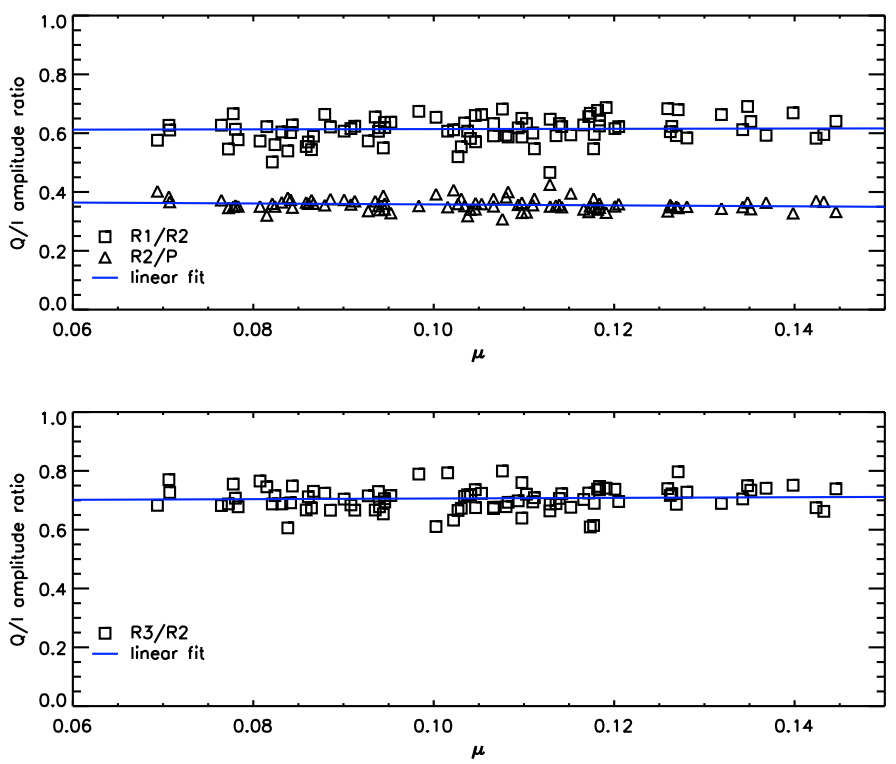

Fig. 8. $Q / I$ amplitude ratios vs. $\mu$ for a smaller range of $\mu$ (close-up of Fig. 7). Here, the solid lines are linear fits through the data points to show nearly constant averages of the ratios with height in the solar atmosphere.

may be increasing, and $R_{1} / R_{2}$ appears constant except the measurement points at $\mu=0.4$, where the signal-to-noise ratio is about six times lower than at $\mu=0.1$ and the error in the ratios amounts to \pm 0.05 . More precise measurements are needed for detecting smaller variations. Generally, the magnetic field strength decreases with height in the solar atmosphere, so one would expect a decrease of $R_{1} / R_{2}$ and $R_{3} / R_{2}$ and an increase of $R_{2} / P$ with $\mu$ (Berdyugina \& Fluri 2004). These variations would only be visible if the magnetic field varies noticeably within the depth of the molecular line forming layer $(\sim 100 \mathrm{~km})$. They could be further influenced by the height dependence of the collision rates and perhaps by depolarization of the nearby Fe I line.

Figure 8 represents a close-up of Fig. 7 for the measurements between $\mu=0.06$ and $\mu=0.15$. Here, the solid lines denote linear fits through the data points. As the lines are nearly horizontal, this confirms our conclusion on the lack of systematic variations larger than 0.04 .

\subsection{Variations with the solar cycle}

Here we compare measurements of the $\mathrm{C}_{2} Q / I$ amplitude ratios obtained in different years of the solar cycle 23 and address their evolution with time. The discussed amplitude ratios are given in Table 2 and are also plotted in Fig. 10.

The earliest observations were taken during the solar maximum at the end of 1999 (sometime between October 1999 and January 2000) and were published in the second solar spectrum atlas (Gandorfer 2000). Although the atlas spectrum is noisier than our new synoptic data, its amplitude ratios significantly deviate from our averages, and this cannot be explained by noise. The only measurement similar to the atlas is $\mathrm{ms}_{080509}$, which was pointed out in Sect. 4.1.

Figure 9 shows an average observation of the synoptic 2007-2009 data (solid), the atlas spectrum (dashed) and the $\mathrm{ms}_{080509}$ measurement (dotted) in $Q / I, I / I_{\mathrm{c}}$ and $V / I$ (the latter is not available for the atlas). Stokes $Q / I$ was smoothed by a threepixel boxcar to reduce the noise. One can see that $V / I$ and $I / I_{\mathrm{c}}$

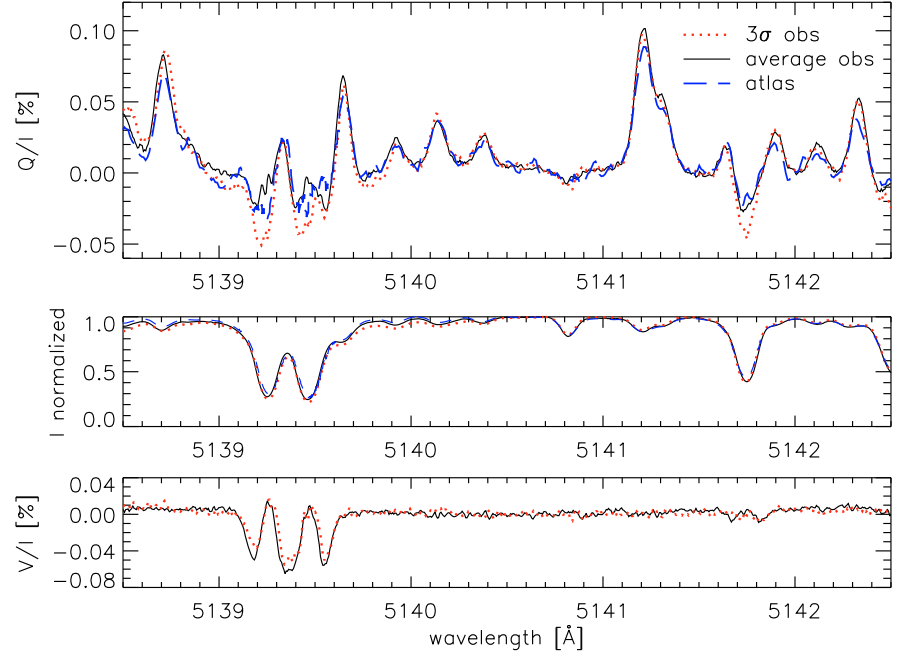

Fig. 9. Stokes $Q / I, I$ and $V / I$ for the average synoptic spectrum (solid), the atlas of the second solar spectrum (dashed) and the single deviating measurement $\mathrm{ms}_{080509}$ (dotted). While $V / I$ and $I$ look similar for all measurements, significant differences are visible in $Q / I$ (see discussion in the text).

are similar for all measurements. The $\mathrm{ms}_{080509}$ shows a small decrease in $I / I_{\mathrm{c}}$ toward the blue, which was most probably caused by vignetting and was not corrected by a flat-field as explained in Sect. 2. There are several differences visible in $Q / I$. The atlas was recorded at a slightly larger limb distance (difference $\Delta \mu=0.01)$ than the other two observations. Therefore, its amplitudes are generally lower, with the exception of the $R_{2}$ peak that reaches the same value as in the synoptic average spectrum. However, the $R_{2}$ peak of $\mathrm{ms}_{080509}$ is significantly higher. The $R_{1}$ amplitude is higher for the average observation than for the atlas and $\mathrm{ms}_{080509}$, and the latter two are similar to each other. Also noticeable is a higher depolarization of $\mathrm{ms}_{080509}$ in the Fe I lines at 5139.2, 5139.4 and 5141.7 $\AA$. This may have caused the lower $R_{1}$ amplitude, which perhaps would otherwise be similar to that of the average spectrum, as is seen for the $R_{3}$ peak. The $P$-triplet amplitudes of $\mathrm{ms}_{080509}$ are intermediate to those of the atlas and the average spectrum. Because the amplitude ratios do not vary with the limb distance, it is easy to see that they are quite similar for the atlas and $\mathrm{ms}_{080509}$ and are systematically different from the average spectrum of 2007-2009, well above the noise level of $5 \times 10^{-5}$.

We have computed half-year averages for the IRSOL observations of 2008-2009, which are plotted in Fig. 10. Because individual measurements of this set are more precise and the overall number of measurements is larger, the averages have smaller error bars. Their values are significantly different from those of the atlas. As we show in Sect. 5.1, a plausible explanation of this difference is that the mean strength of a turbulent magnetic field was varying with the solar activity level, and we may expect more measurements similar to $\mathrm{ms}_{080509}$ as the cycle 24 progresses.

The amplitude ratios from the THEMIS 2008 observations do not show variations among the eight recorded position angles. So, when averaged, they are equal to the synoptic IRSOL measurements within the error bars. Note that the ratios do not depend on the telescope.

The gap between the atlas and the synoptic data, i.e. during the declining phase of the solar cycle, is covered by other data sets. Two measurements were taken at IRSOL in 2003. Their 


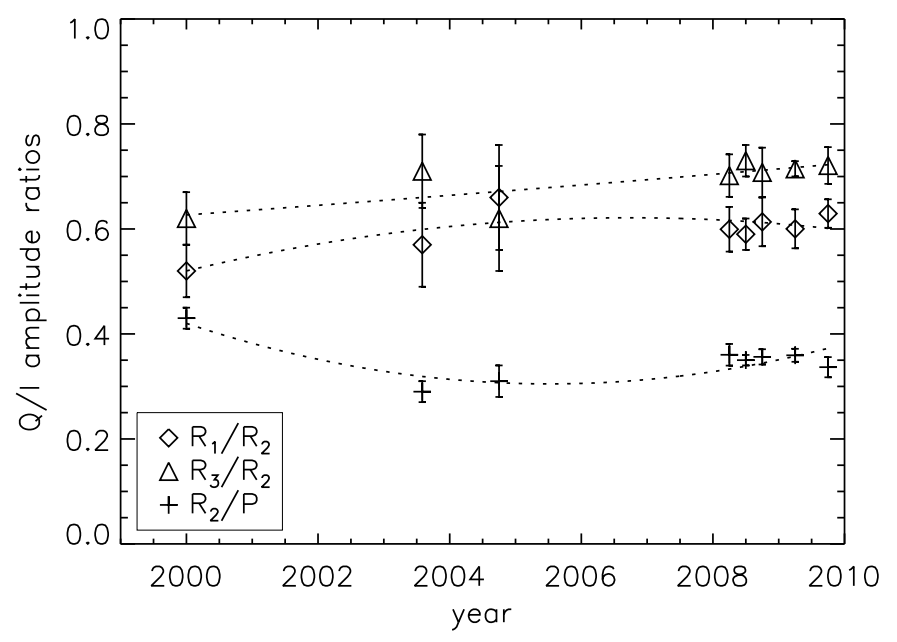

Fig. 10. Amplitude ratios for all analyzed data sets. For the synoptic measurements, the calculated ratios were binned in half-year intervals. A systematic decrease of $R_{2} / P$ and increase of $R_{1} / R_{2}$ and $R_{3} / R_{2}$ seem to occur as the solar activity declines. Second-order polynomial fits to the line ratios are shown with dotted lines.

noise level was comparable to that of the atlas, and their amplitude ratios were obtained with larger errors as compared to the synoptic amplitude ratios. While the value of $R_{1} / R_{2}$ lies between the atlas and synoptic measurements, $R_{2} / P$ is the lowest among all. However, on average, these measurements are closer to the synoptic data than to the atlas. The six ratios from the THEMIS/MTR observations in 2004 have the largest errors because of the problem with fringes. However, within their error bars, the ratios are similar to all other observations and close to those of IRSOL 2003. Thus, despite their large errors, these two data sets are very valuable because they provide a constraint on the magnetic field evolution. They clearly indicate that no drastic changes occurred in the intermediate years. When the amplitudes of the two sets are averaged, one obtains for the years 2003-2004: $R_{1} / R_{2}=0.62 \pm 0.10, R_{3} / R_{2}=0.67 \pm 0.09$ and $R_{2} / P=0.30 \pm 0.03$. These are closer to the synoptic data than to the atlas, as can also be seen in Fig. 10.

To investigate the significance of the variations of these amplitude ratios we calculated the F-statistic (Press et al. 2002). The null hypothesis was that the ratios were constant over time. This was tested against the possibility that the ratios vary as polynomials, in particular, that $R_{1} / R_{2}$ and $R_{3} / R_{2}$ anticorrelates with $R_{2} / P$, as would be expected for variations caused by the magnetic field. Least-squares fits to the data points in Fig. 10 were performed for constant functions (not shown) and second-order polynomials (dotted). The residuals of the fits to the three ratios together weighted with the individual errors were used as input for the F-statistic. The result was that the variances of the two fits are different at the $3 \sigma$ level. It is therefore probable that the turbulent magnetic field varied during the solar cycle. However, this conclusion is strongly influenced by the measurement in 2000 at the solar maximum, which should be verified with new observations. Our synoptic program will be continued and, if this variation is real, we should be able to detect it during the ascending phase of the solar cycle 24, unless it is much weaker than the cycle 23 .

We conclude that mild variations of the amplitude ratios occurred during the second half of the cycle 23 . While the $R_{1} / R_{2}$ and $R_{3} / R_{2}$ ratios were apparently increasing, the $R_{2} / P$ was

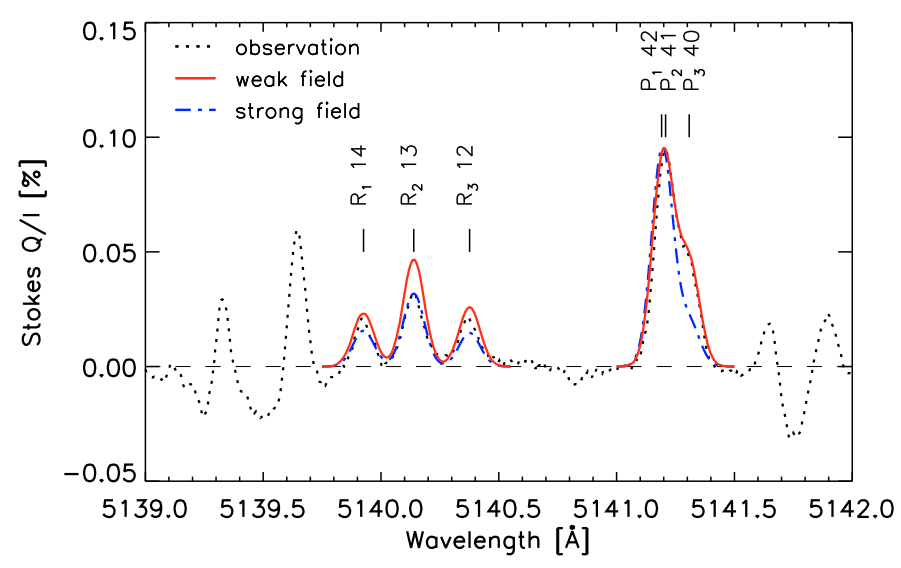

Fig. 11. Two fits (solid and dash-dotted) to the synoptic observation (dotted) of Fig. 1 (averaged along the slit) using the differential Hanle model. The profiles shown with the solid line were calculated with a magnetic field of $7 \mathrm{G}$ and those shown with the dash-dotted line represent $90 \mathrm{G}$, both with different scaling factors $q$. There is apparently no unique set of parameters fitting all lines of both triplets simultaneously.

decreasing. This behavior is a signature of weakening turbulent magnetic fields, as shown in Sect. 5.1.

\section{Modeling of the data}

\subsection{Modeling of Stokes $Q / I$}

Here we employ the differential Hanle effect model described in Sect. 3. We apply it to the average synoptic data and to the earlier measurements to interpret the observed Stokes $Q / I$ amplitude ratios in terms of the turbulent magnetic field strength.

Figure 11 shows two example fits (solid and dash-dotted) to the observation (dotted) shown in Fig. 1. The two fits differ only in $B$ and $q$ (assuming $\alpha=1$ ). The solid line fit was created for a magnetic field of $7 \mathrm{G}$ and for the $q$ that result in the best fit to the $P$-triplet. It is obvious that while the lines of the $P$-triplet are fitted well, the amplitude of the whole $R$-triplet is too high. The same model, with only slightly adjusted $q$ to account for a small difference in heliocentric angle $\mu$, provided a better fit to the second solar spectrum atlas, where the $R_{2} / P$ ratio was higher and the $R_{1} / R_{2}$ and $R_{3} / R_{2}$ ratios were lower. The fit was not perfect, but the noise was higher too. Note that this is the same model as in Berdyugina \& Fluri (2004) except for a factor of two lower Einstein coefficients, which leads to the factor of two weaker field. Since the measurement presented in Fig. 11 is quite typical for our synoptic program as concerns the line ratios, it is clear that the atlas measurement is rather unique as discussed in Sect. 4, and we should look for a reason for this difference.

A lower $R_{2} / P$ ratio and higher $R_{1} / R_{2}$ and $R_{3} / R_{2}$ ratios may indicate a stronger magnetic field (Berdyugina \& Fluri 2004). Therefore, we searched for the best-fit model with a magnetic field of $\geq 80 \mathrm{G}$. The result is shown with the dash-dotted line in Fig. 11. With such a field strength we obtained perfect fits to $P_{1}+P_{2}$ and $R_{2}$, but $R_{1}, R_{3}$ and especially $P_{3}$ are unrealistically depolarized. We can exclude unaccounted blends at these wavelengths because all nearby triplets with similar quantum numbers show the same effect at these fields. Thus, a strong field of $\geq 80 \mathrm{G}$ can be definitely excluded.

It appears more feasible that the polarization is formed at weaker magnetic fields, largely because of $P_{3}$, and there is a difference between the two triplets, unaccounted for in the present modeling. One possibility is the depolarization of the continuum 


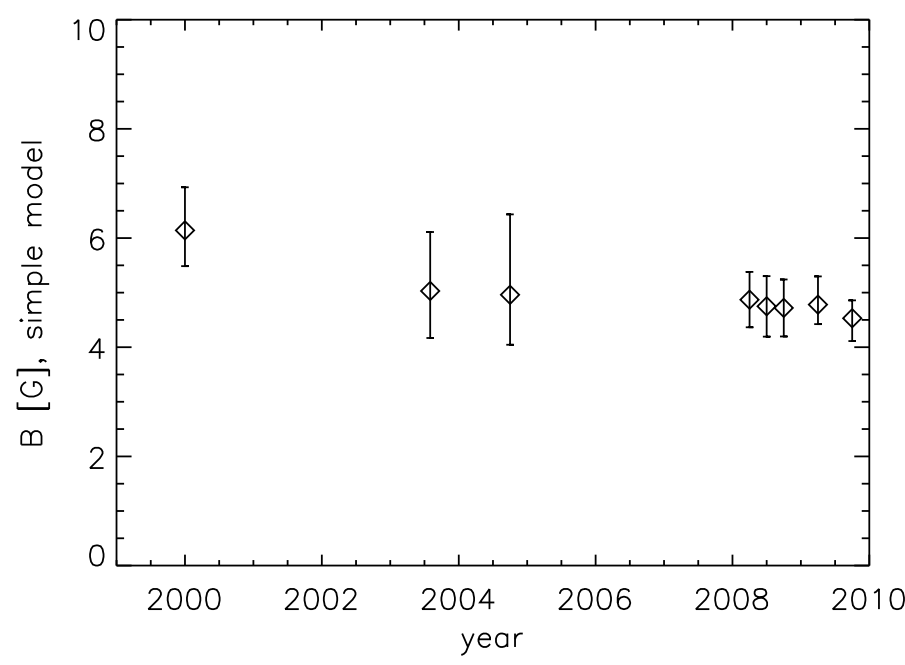

Fig. 12. Magnetic field strength calculated with the model described in Sect. 3 applied to the $R$-triplet ratios only. The data sets are the same as in Fig. 10.

owing to line opacity, which is certainly larger for the $P$-triplet. Since the $\mathrm{C}_{2}$ polarization is formed higher in the atmosphere than that of the continuum, this implies that line polarization is built on top of a depolarized profile (Fluri \& Stenflo 2003), which reduces the peak polarization with respect to the local, unaffected continuum. However, these lines are still optically thin, so the difference in their optical thicknesses is not sufficient to explain the difference between modeling and observation. This effect is taken into account in the radiative transfer modeling in Paper II and confirmed to be very small. Another possibility is depolarization owing to collisions. There are indications that collision rates depend on the total angular momentum $J$ and can be higher for lower $J$ numbers (Derouich 2006). In this case, the depolarization would be stronger for the $R$-triplet than for the $P$-triplet, which is in accord with our measurements. This effect is also accounted for in Paper II.

If the magnetic filling factor $\alpha$ was not fixed, then the results were qualitatively similar. Good fits for all ratios were obtained for $\alpha \approx 0.8$ and $60 \leq B \leq 90 \mathrm{G}$ (depending on the measurement). The measured $R_{2} / P$ ratio can only be obtained for higher field strengths, as can be seen in Fig. 3. At these field strengths $P_{3}$, which was not taken into account in the line ratios, is already significantly depolarized. This implies that the magnetic field must be below $60 \mathrm{G}$, and the best fit then yields $\alpha=1.0$ and $3 \leq B \leq 8 \mathrm{G}$. This also supports the conclusion that there seems to be a significant difference between the two triplets. It also confirms a high filling factor for turbulent magnetic fields.

Nevertheless, it is possible to evaluate the turbulent magnetic field strength from the amplitude ratios in the $R$ - and $P$-triplets separately with the differential Hanle effect model in the weakfield regime, because the $J$-dependent effects will be strongly diminished within one triplet. Using the theoretical ratios as functions of the magnetic field strength shown in Fig. 3, we interpret the measured ratios presented in Fig. 10 in terms of $B$, keeping the filling factor as a free parameter. The result yields $\alpha=1$ for all fits and is shown in Fig. 12.

We conclude that the average strength of the turbulent magnetic field during the solar minimum in 2008-2009 was at least $4.7 \pm 0.2 \mathrm{G}$. It may have reduced in strength since the solar maximum in 2000, for which the single measurement indicated the field strength of at least $6 \pm 1 \mathrm{G}$. The strength for intermediate years (2003-2004) is $5 \pm 1 \mathrm{G}$.

Thus, the magnetic field may have weakened by a few Gauss as the solar activity changed from maximum to minimum during the last decade. Note that the scale of this variation is still modeldependent. In the present interpretation we neglected some depolarizing effects, e.g. collisions. This provides the robust lower limits on both the average strength and the variation scale of the turbulent magnetic field (as indicated above). When these effects are more consistently accounted for, the strength of the field and its variations scale up. For instance, we show in Paper II that the scaling factor may be as large as two, which leads to fields weaker than $\sim 10 \mathrm{G}$. This is still within the range of previously reported values inferred from molecular lines and significantly lower than those for atomic lines. To resolve this contradiction, one needs to apply the differential Hanle effect to atomic lines and, therefore, reduce the model dependent effects. Our synoptic observations have a potential for this analysis and we will pursue it in the near future.

\subsection{Modeling of Stokes V/I}

The signals in Stokes $V / I$ can be used to calculate the line-ofsight (LOS) magnetic field component. Several measurements taken under good seeing conditions show $V / I$ signals on the order of $0.1 \%$ in the Fe I lines. The magnetic patches of the same polarity have a size of 3 to 10 pixels along the slit, equal to $4.4^{\prime \prime}$ to $14.6^{\prime \prime}$ with the width of $0.5^{\prime \prime}$. Because the signal-to-noise ratio is sufficiently high, we do not need to average $V / I$ over the whole slit like $Q / I$, but it is sufficient to average several pixels.

Using the STOPRO code (Solanki 1987; Frutiger et al. 2000) we calculated Stokes $V / I$ profiles for the three iron lines next to the $\mathrm{C}_{2}$ lines. The synthetic profiles were broadened using an instrumental profile of $50 \mathrm{~m} \AA$. Because the strong iron lines form in NLTE conditions, we slightly adjusted their oscillator strengths, so that the calculated Stokes $I$ better matches the observations. Unfortunately, the available set of iron lines did not allow us to separate the filling factor and the field strength. Therefore, we could determine only their product, so the field strengths discussed below are actually low limits and correspond to the filling factor $\alpha=1$. Because the spatial resolution was low, we could expect cancellations of Stokes $V$ within the observed patches.

Figure 13 shows the best fit for a magnetic field of $5 \pm 1 \mathrm{G}$ (solid thick lines) to an observation of August 16, 2008, averaged over $5.8^{\prime \prime}$ (dotted). The thin lines are calculations for $3 \mathrm{G}$ and $7 \mathrm{G}$ to demonstrate the sensitivity of the $V / I$ amplitudes to the weak magnetic field. The intensity decrease in Stokes $I$ at longer wavelengths in this spectrum results from vignetting effects and cannot be completely removed because of the absence of spectral flatfields. Only a first order correction was applied to Stokes $I$ by fitting a second-order polynomial through continuum points. As mentioned before, Stokes $I$ should be treated with caution, but $V / I$ does not suffer from any vignetting or gain table effects.

Fits to different observations on various days and for different parts of the slit revealed magnetic fields of $3 \mathrm{G}$ to $8 \mathrm{G}$ with the errors of $\pm 1 \mathrm{G}$. Weaker magnetic fields could not be accurately determined because of the noise level. Despite measuring only $\alpha B$, we can exclude strong fields with a high filling factor, because they would contribute to $Q / I$ due to the transverse Zeeman effect. While there were some small variations visible in $Q / I$ of the iron lines across the slit, the signals never exceeded the level of the scattering polarization $(\max 0.1 \%)$, and 

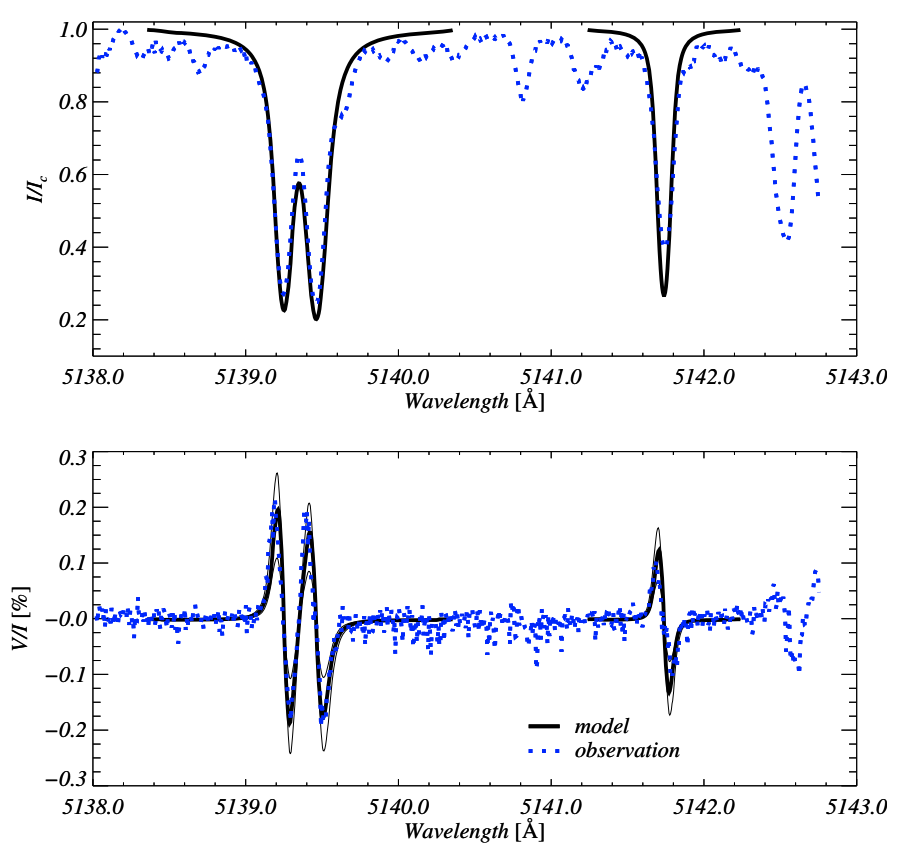

Fig. 13. One of the observed Stokes $I / I_{\mathrm{c}}$ and $V / I$ spectra recorded near the heliospheric south pole on August 16, 2008, and averaged over four pixels, corresponding to 5.8" (dotted). Modeled Fe I profiles are shown for a magnetic field of $5 \mathrm{G}$ (thick solid lines) as well as for $3 \mathrm{G}$ and $7 \mathrm{G}$ (thin lines) for comparison.

no clear transverse Zeeman signatures could be seen. Modeling with STOPRO indicates that a $Q / I$ amplitude in the range of $0.1 \%$ in the iron line at $5139.2 \AA$ can be expected for a purely transversal field of $50 \mathrm{G}$. We therefore conclude that average magnetic fields $(\alpha B)$ transverse to our line of sight (vertical with respect to the solar surface) must be below $50 \mathrm{G}$ for our spatial and temporal resolution.

To classify the occurrence of the $V / I$ amplitudes, we analyzed 16 measurements with sufficiently good seeing (well visible $V / I$ pattern along the slit, similar to Fig. 1). Every two pixels along the slit $\left(0.5^{\prime \prime} \times 2.9^{\prime \prime}\right)$ were averaged for a higher signal-to-noise ratio profile. A STOPRO model profile was then fitted to each of the observed profiles, in order to determine the $V / I$ amplitude. Figure 14 shows a histogram of the V/I amplitude of the blue lobe of the Fe I 5139.2 $\AA$ line. A total of more than 1000 data points were used. The average noise level of the observations used to determine the histogram data points is $2.6 \times 10^{-5}=2.6 \times 10^{-3} \%$. The distribution is close to normal with a full width at half maximum (FWHM) of $3 \mathrm{G}$, reaching maximum values of $8 \mathrm{G}$. We compare it to the results of other authors in Sect. 6.2.

\section{Discussion and conclusion}

\subsection{Magnetic fields inferred from the Hanle effect}

We have presented a synoptic program for monitoring spatial and temporal variations of the turbulent magnetic field. A total of 78 Stokes $Q / I$ measurements of the $\mathrm{C}_{2}$ lines at $5141 \AA$ were analyzed by means of the differential Hanle effect. We inferred observed amplitude ratios of several lines that are sensitive to magnetic fields and investigated their behavior. The resulting ratios did not show any variations during the past two years of the solar minimum, independently of the observed position angle on the Sun. We therefore conclude that the turbulent magnetic field

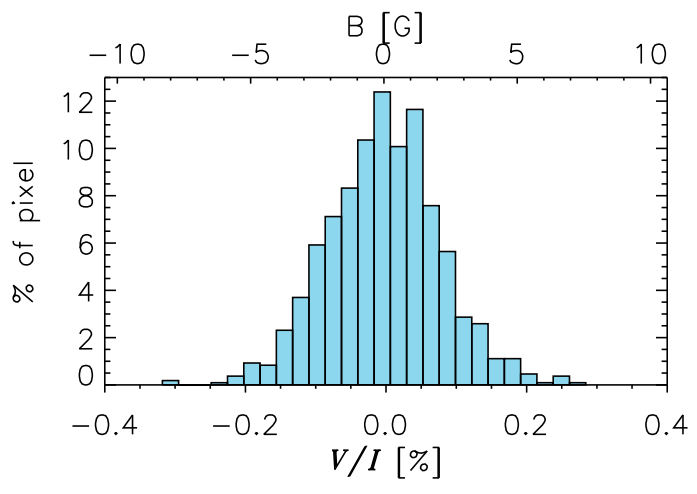

Fig. 14. Distribution of observed $V / I$ amplitudes of the Fe I $5139.2 \AA$ line averaged within $0.5^{\prime \prime} \times 2.9^{\prime \prime}$ patches along the slit.

has been surprisingly spatially homogeneous and temporally invariant for the past two years when measured using the $C_{2}$ lines. The lower limit for the average strength of this field of $4.7 \pm 0.2 \mathrm{G}$ was evaluated.

A comparison with the earlier measurements in 2000 and 2003-2004 indicated that the mean strength of the turbulent field has probably mildly decreased ( $3 \sigma$-limit) during the descending phase of the solar activity. It appears that the average strength of the turbulent field at the maximum of the solar activity in 2000 was about 5-7 G, i.e. somewhat higher than in 2007-2009. However, the statistics and quality of the earlier data are unfortunately poor and more frequent observations as in our synoptic program are needed to reveal the correlation at a more significant level. We may expect more prominent changes during the ascending phase of the solar cycle because it occurs on a shorter time scale.

\subsection{Magnetic fields inferred from the Zeeman effect}

Our results from Fe I Stokes $V / I$ show a nearly horizontal flux of 3 to $8 \mathrm{Mx} \mathrm{cm}^{-2}$ within about $0.5^{\prime \prime} \times 5^{\prime \prime}$ patches along the slit. This value agrees well with those of other authors apart from the horizontal flux discovered by Lites et al. (2008), which is significantly higher. However, those authors measured it at the disk center with a higher spatial resolution $\left(0.3^{\prime \prime}\right)$ and using different Fe I lines. Therefore, differences can be expected and it is worth to take additional ZIMPOL measurements - for example full Stokes data at the disk center or high spatial resolution scattering polarization observation - to be able to make a more thorough comparison with small-scale magnetic fields seen in Hinode data.

It is interesting that both the Hanle and Zeeman effects in two different types of lines (molecular $\mathrm{C}_{2}$ and atomic $\mathrm{Fe} \mathrm{I}$ ) result in similar field strengths. The average strength of $4.7 \pm 0.2 \mathrm{G}$ inferred from the differential Hanle effect does not depend on the filling factor and gives us the field strength of the turbulent, unresolved fields. Stokes $V / I$ however describes resolved fields of $3 \mathrm{G}$ to $8 \mathrm{G}$. One can imagine two simple geometric scenarios, both resulting in the observed $V / I$ :

1) the magnetic fields responsible for the Hanle effect are nearly resolved and are distributed along the slit as indicated by the patches observed in $V / I$. This implies a low average field strength and a high filling factor within the patches;

2) weak, unresolved, turbulent fields are interspersed with occasional flux tubes or patches of a stronger magnetic field. 
Table 3. A summary of quiet Sun magnetic field measurements.

\begin{tabular}{lcccccc}
\hline \hline Solar region & Instrument & Lines & Spatial resol. & $\alpha B\left[\mathrm{Mx} \mathrm{cm}^{-2}\right]^{a}$ & Method & Reference \\
\hline limb & ZIMPOL II & $\mathrm{C}_{2} 5141$ & $0.5^{\prime \prime} \times 180^{\prime \prime}$ & $B_{\mathrm{t}}=4.7 \pm 0.2[\mathrm{G}](\alpha=1)$ & diff. Hanle & this paper \\
limb & ZIMPOL II & Fe I 5139 & $0.5^{\prime \prime} \times \approx 5^{\prime \prime}$ & $B_{\mathrm{h}}=3-8 ; B_{\mathrm{v}}<50$ & $V / I$ & this paper \\
disk center & ASP & Fe I 6301/2 & $1^{\prime \prime} \times 1^{\prime \prime}$ & $B_{\mathrm{v}}=\{18.6(\mathrm{QS}), 10.7(\mathrm{IN})\}$ & $Q / I, U / I, V / I$ & Lites (2002) \\
disk center & VTT/GFPI & Fe I 6301/2 & $0.5^{\prime \prime} \times 0.5^{\prime \prime}$ & $B_{\mathrm{v}}=20$ & $V / I$ & Domínguez Cerdeña et al. (2003) \\
disk center & DLSP & Fe I 6301/2 & $0.6^{\prime \prime} \times 0.6^{\prime \prime}$ & $B_{\mathrm{v}}=\{12.5(\mathrm{QS}), 8.8(\mathrm{IN})\}$ & $Q / I, U / I, V / I$ & Lites \& Socas-Navarro (2004) \\
disk center & HINODE/SP & Fe I 6301/2 & $0.3^{\prime \prime} \times 0.3^{\prime \prime}$ & $B_{\mathrm{v}}=\{16.7(\mathrm{QS}), 8.4(\mathrm{IN})\}$ & $Q / I, U / I, V / I$ & Orozco Suárez et al. (2007) \\
& & & & $B_{\mathrm{v}}=9.5, B_{\mathrm{h}}=11.3$ & & \\
disk (limb) & HINODE/SP Fe I 6301/2 & $0.3^{\prime \prime} \times 0.3^{\prime \prime}$ & $B_{\mathrm{v}}=11, B_{\mathrm{h}}=55$ & $Q / I, U / I, V / I$ & Lites et al. (2008) \\
S pole & HINODE/SP Fe I 6301/2 & $0.3^{\prime \prime} \times 0.3^{\prime \prime}$ & $>10[\mathrm{G}](\alpha=1)$ & $Q / I, U / I, V / I$ & Tsuneta et al. (2008) \\
disk & HINODE/SP Fe I 6301/2 & $0.3^{\prime \prime} \times 0.3^{\prime \prime}$ & bimodal & $Q / I, U / I, V / I$ & Stenflo (2010) \\
& & & & $B_{\mathrm{v}}=\{5-10 \mathrm{G} ; 1 \mathrm{kG}\}$ & & \\
\hline
\end{tabular}

(a) $B_{\mathrm{t}}=$ turbulent field, $B_{\mathrm{h}}=$ horizontal field, $B_{\mathrm{v}}=$ vertical field.

This implies that our observed $V / I$ is composed of structures of different filling factors and field strengths.

Our observations cannot distinguish between these two cases. However, the first scenario contradicts higher spatial resolution observations, which show a large difference between magnetic fluxes densities in network and internetwork (IN) at a resolution scale nearly one magnitude better than ours. Thus, the second scenario is more probable.

The fields observed in $V / I$ are nearly horizontal with an angle of about $6^{\circ}$ relative to the local horizontal direction for $\mu=0.1$. The magnetic flux of our average single-polarity patch, $5 \mathrm{G} \times$ $0.5^{\prime \prime} \times 5.8^{\prime \prime}$, amounts to $7.9 \times 10^{16} \mathrm{Mx}$. The size of this patch roughly corresponds to a mesogranular scale on the Sun. Our spatial ( $\left.2^{\prime \prime}\right)$ and temporal (30 min) resolutions suggest that the field within this structure is predominantly of the same polarity, but the existence of $\mathrm{kG}$ fields found in the quiet Sun at higher resolution (Domínguez Cerdeña et al. 2003; Orozco Suárez et al. 2007) cannot be excluded.

The comparison of our results with those of others is not straightforward. The magnetic flux is dependent on the observed resolution and therefore on the telescope and instrument. The recovery of the apparent flux and the intrinsic field strength is generally performed with various methods, for example through inversions, the weak field approximation or through a calibration of the wavelength integrated polarization signal (Lites \& SocasNavarro 2004; Lites et al. 2008). All studies used different Fe I lines (namely 6301.5 and $6302.5 \AA$ ) from ours, and a possible height dependency of the magnetic field is not known. A summary of some results of different authors and instruments used for our comparison can be found in Table 3 .

Domínguez Cerdeña et al. (2003) found an unsigned magnetic field of approximately $20 \mathrm{G}$, which is lowered to 7-9 G when their observations with a resolution of $0.5^{\prime \prime}$ are artificially degraded to a $1^{\prime \prime}$ resolution. Other authors (see Lites \& Socas-Navarro 2004) find no change of the field strength when the resolution is changed. Using the DLSP, an instrument with $0.6^{\prime \prime}$ resolution, Lites \& Socas-Navarro (2004) found an apparent flux density of $8 \mathrm{G}$ to $10 \mathrm{G}$ in the IN for different maps. They found no increase in the net unsigned flux compared to previous, lower resolution, observations. This behavior resembles the selfsimilarity of the distribution of the solar magnetic fields (Stenflo $\&$ Holzreuter 2003). These values agree with our result from the Hanle effect and could also be true for the Zeeman observations, assuming a high filling factor. However, all these observations used measurements that were taken close to disk center, and $V / I$ is then the locally vertical flux.
Only recent observations using HINODE distinguish locally horizontal and vertical flux. Orozco Suárez et al. (2007) find an unsigned apparent flux density of $9.5 \mathrm{Mx} \mathrm{cm} \mathrm{cm}^{-2}$ and $11.3 \mathrm{Mx} \mathrm{cm}^{-2}$ for the vertical and horizontal flux, respectively, which is close to our results. Also, the result of Tsuneta et al. (2008), who conclude that for a uniformly distributed polar field they would estimate an effective field strength of $10 \mathrm{G}$, agrees well with our average field. Stenflo (2010) finds two populations of magnetic field strengths with the lower one resembling our histogram very well, also in its FWHM. Furthermore, they conclude on similar values for horizontal and vertical fields, in agreement with our results.

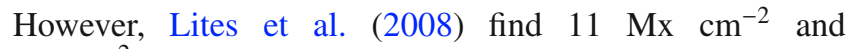
$55 \mathrm{Mx} \mathrm{cm}^{-2}$ for the fluxes, i.e. a rather large horizontal apparent flux density, which we do not observe. These values are derived from measurements taken at disk center. They also show an interesting measurement close to the limb (their Fig. 14), where it is evident that Stokes $V / I$ appears to decrease toward the limb, exactly opposite to what may be expected at first from their findings at disk center. Our result agrees with the small $V / I$ close to the limb, and several explanations can be considered. It is possible that either the magnetic field decreases with height (closer to the limb) or that cancellation effects get larger because of the geometry of the small-scale magnetic field distribution or that the calibration at disk center was not perfect and their $B_{\text {app }}^{\mathrm{T}}$ may not be interpreted in terms of resolved horizontal field strength. A more detailed analysis would certainly be interesting, both by taking full Stokes ZIMPOL data at disk center and inverting the Stokes parameters and by analyzing HINODE data taken at the limb, with a higher $\mathrm{S} / \mathrm{N}$, for example through spatial and temporal averaging.

Acknowledgements. We gratefully acknowledge the technical support provided by Daniel Gisler, Peter Steiner and Renzo Ramelli. This work has been funded by the SNSF, grants 200020-117821 and 200020-127329. S.V.B. acknowledges the EURYI (European Young Investigator) Award provided by the ESF (see www.esf.org/euryi) and the SNSF grant PE002-104552. THEMIS is operated on the Island of Tenerife by the Centre National de la Recherche Scientifique (CNRS, France), the authors are grateful to B. Gelly and C. Le Men for optimizing the interface to install ZIMPOL.

THEMIS data have been provided by BASS2000. We are very grateful to Jean Arnaud for letting us use his data-reduction routines for the MTR THEMIS data.

\section{References}

Allen, C. W. 1973, Astrophysical quantities (London: Athlone Press) Asensio Ramos, A., \& Trujillo Bueno, J. 2007, Mem. Soc. Astron. Ital., 78, 42 Berdyugina, S. V., \& Fluri, D. M. 2004, A\&A, 417, 775 
Berdyugina, S. V., \& Solanki, S. K. 2002, A\&A, 385, 701

Berdyugina, S. V., Stenflo, J. O., \& Gandorfer, A. 2002, A\&A, 388, 1062

Bommier, V., \& Molodij, G. 2002, A\&A, 381, 241

Bommier, V., \& Rayrole, J. 2002, A\&A, 381, 227

Bommier, V., Landi Degl'Innocenti, E., Feautrier, N., \& Molodij, G. 2006, A\&A, 458, 625

Derouich, M. 2006, A\&A, 449, 1

Derouich, M., Bommier, V., Malherbe, J. M., \& Landi Degl'Innocenti, E. 2006, A\&A, 457, 1047

Domínguez Cerdeña, I., Kneer, F., \& Sánchez Almeida, J. 2003, ApJ, 582, L55

Faurobert, M., \& Arnaud, J. 2003, A\&A, 412, 555

Faurobert, M., Arnaud, J., Vigneau, J., \& Frisch, H. 2001, A\&A, 378, 627

Feller, A. J. 2007, Ph.D. Thesis, ETH, Zurich, Switzerland

Fluri, D. M., \& Stenflo, J. O. 1999, A\&A, 341, 902

Fluri, D. M., \& Stenflo, J. O. 2003, A\&A, 398, 763

Frutiger, C., Solanki, S. K., Fligge, M., \& Bruls, J. H. M. J. 2000, A\&A, 358, 1109

Gandorfer, A. 2000, The Second Solar Spectrum, Vol I: $4625 \AA$ to $6995 \AA$ (Zurich: $\mathrm{VdF}$ )

Gandorfer, A. M., Povel, H. P., Steiner, P., et al. 2004, A\&A, 422, 703

Gisler, D. 2005, Ph.D. Thesis, ETH, Zurich, Switzerland

Ivanov, V. V. 1991, in Stellar Atmospheres: Beyond Classical Models, NATO

ASI Series C 341, ed. L. Crivellari, I. Hubeny, \& D. G. Hummer (Dordrecht: Kluwer), 81

Kleint, L., Feller, A., \& Bianda, M. 2008, in 7014, Ground-based and Airborne Instrumentation for Astronomy II, ed. I. McLean, \& M. Casali, Proc. SPIE, 701414

Kleint, L., Berdyugina, S. V., Shapiro, A. I., \& Bianda, M. 2010a, in SOHO-23: Understanding a Peculiar Solar Minimum, ed. S. Cranmer, T. Hoeksema, \& J. Kohl, ASP Conf. Ser., 428, 103

Kleint, L., Shapiro, A. I., Berdyugina, S. V., \& Bianda, M. 2010b, A\&A, submitted
Kurucz, R. L., Furenlid, I., Brault, J., \& Testerman, L. 1984, Solar flux atlas from 296 to $1300 \mathrm{~nm}$, ed. R. L. Kurucz, I. Furenlid, J. Brault, \& L. Testerman Lites, B. W. 2002, ApJ, 573, 431

Lites, B. W., \& Socas-Navarro, H. 2004, ApJ, 613, 600

Lites, B. W., Kubo, M., Socas-Navarro, H., et al. 2008, ApJ, 672, 1237

Orozco Suárez, D., Bellot Rubio, L. R., del Toro Iniesta, J. C., et al. 2007, ApJ, 670, L61

Press, W. H., Teukolsky, S. A., Vetterling, W. T., \& Flannery, B. P. 2002, Numerical recipes in $\mathrm{C}++$ : the art of scientific computing, ed. W. H. Press, S. A. Teukolsky, W. T. Vetterling, \& B. P. Flannery

Solanki, S. K. 1987, Ph.D. Thesis, Ph.D. Thesis, ETH, Zürich, Switzerland

Stenflo, J. O. 1994, Solar Magnetic Fields (Dordrecht: Kluwer)

Stenflo, J. O. 2003, in Solar Polarization, Proc. 3rd SPW, ed. J. Trujillo Bueno, \& J. Sánchez Almeida, ASP Conf. Ser., 307, 385

Stenflo, J. O. 2006, in Solar Polarization, Proc. 4th SPW, ed. R. Casini, \& B. W. Lites, ASP Conf. Ser., 358, 215

Stenflo, J. O. 2010, A\&A, 517, A37

Stenflo, J. O., \& Keller, C. U. 1997, A\&A, 321, 927

Stenflo, J. O., \& Holzreuter, R. 2003, in Current Theoretical Models and Future High Resolution Solar Observations: Preparing for ATST, ed. H. SawayaLacoste, ASP Conf. Ser., 286, 169

Stenflo, J. O., Bianda, M., Keller, C. U., \& Solanki, S. K. 1997, A\&A, 322, 985

Stenflo, J. O., Keller, C. U., \& Gandorfer, A. 1998, A\&A, 329, 319

Trujillo Bueno, J. 2003, in Solar Polarization 3, ed. J. Trujillo Bueno, \& J. Sánchez Almeida, ASP Conf. Ser., 307, 407

Trujillo Bueno, J., Shchukina, N., \& Asensio Ramos, A. 2004, Nature, 430, 326

Trujillo Bueno, J., Asensio Ramos, A., \& Shchukina, N. 2006, in Solar Polarization 4, ed. R. Casini, \& B. W. Lites, ASP Conf. Ser., 358

Tsuneta, S., Ichimoto, K., Katsukawa, Y., et al. 2008, ApJ, 688, 1374 